\title{
Estimating progress of specific deposit in a dual-media BOPS-sand water filter using a matrix approach
}

\begin{abstract}
A new matrix approach which incorporated Rajagopalan and Tien model was developed to simulate the progress of sediment deposition in sublayers of filtration media for mono-media sand and BOPS filters as well as dual-media BOPS-sand filters, at different time until the end of filtration process. The results of specific deposit in the matrix form can be used later to predict the operational head loss in the sublayers of filter at different time. The experiment on filtration process using a filter column was carried out to treat raw water at typical rapid filtration flow rate of 3 to $7.5 \mathrm{~m} / \mathrm{h}$. The influent and effluent water turbidity, total suspended solids and the operational head loss at specific thickness and time were monitored and recorded during filtration process. A dual-media filter of BOPS-sand with ES of 0.8:0.5 mm to $1.0: 0.5 \mathrm{~mm}$ are effective in treating raw water and produce high quality effluent turbidity as good as the conventional mono-media sand filter.
\end{abstract}

Keyword: Specific deposit; Dual-media BOPS-sand filter; Matrix approach 